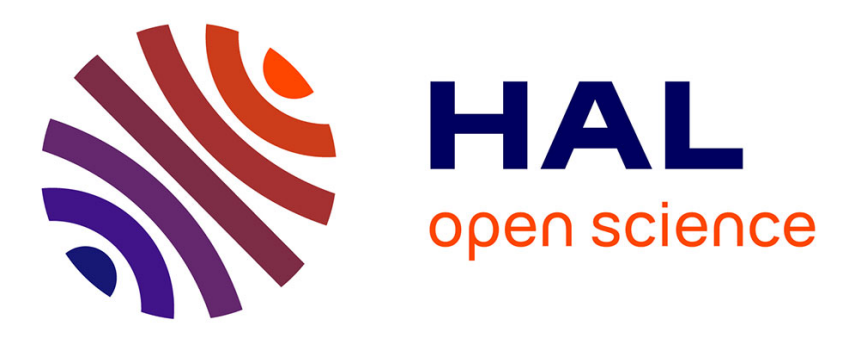

\title{
Internal Mismatch Stresses in Nickel-Based Superalloys : A Finite Element Approach
}

\author{
A. Hazotte, A. Racine, S. Denis
}

\section{To cite this version:}

A. Hazotte, A. Racine, S. Denis. Internal Mismatch Stresses in Nickel-Based Superalloys: A Finite Element Approach. Journal de Physique IV Proceedings, 1996, 06 (C1), pp.C1-119-C1-128. 10.1051/jp4:1996112 . jpa-00254144

\section{HAL Id: jpa-00254144 https://hal.science/jpa-00254144}

Submitted on 1 Jan 1996

HAL is a multi-disciplinary open access archive for the deposit and dissemination of scientific research documents, whether they are published or not. The documents may come from teaching and research institutions in France or abroad, or from public or private research centers.
L'archive ouverte pluridisciplinaire HAL, est destinée au dépôt et à la diffusion de documents scientifiques de niveau recherche, publiés ou non, émanant des établissements d'enseignement et de recherche français ou étrangers, des laboratoires publics ou privés. 


\title{
Internal Mismatch Stresses in Nickel-Based Superalloys: A Finite Element Approach
}

\author{
A. Hazotte, A. Racine and S. Denis \\ Laboratoire de Science et Génie des Matériaux Métalliques, URA 159 du CNRS, Ecole des Mines, Parc de \\ Saurupt, 54042 Nancy cedex, France
}

\begin{abstract}
A three-dimensional finite element (F.E.) model is used to estimate the mismatch stresses at a microscopic scale in $\gamma-\gamma^{\prime}$ single-crystal nickel-based superalloys. F.E. calculations point out the specificity of stress/strain distributions in these alloys, in particular the fact that internal stresses concentrate in the connected $\gamma$ matrix. The analysis of stress/strain fields and elastic energy evolution as a function of precipitate morphology and volume fraction allows to explain some atypical precipitate shape changes such as "splitting" during precipitation treatments or "raftening" during high temperature creep tests.
\end{abstract}

\section{INTRODUCTION}

Nickel-based superalloys used to manufacture single-crystal turbines blades are strengthened by precipitation of a high volume fraction of an intermetallic $\mathrm{Ni}_{3} \mathrm{Al}$-based phase ( $\gamma^{\prime}$ phase) in the Ni-based matrix $(\gamma$ phase). Figure 1 shows their typical microstructure after standard precipitation heat treatment. Both $\gamma$ and $\gamma^{\prime}$ phases have f.c.c. crystallographic lattices. They are in coherence after standard heat treatments, although they present a small (less than $1 \%$ ) difference of parameter. The existence of this lattice mismatch results in internal stresses which strongly influence the high temperature mechanical behaviour of these alloys, as well as their microstructural evolution during service. In particular, these mismatch stresses have been unambiguously related to the occurence of precipitate shape changes during either precipitation or ageing treatments.

Most part of the theoretical studies concerning these internal mismatch stresses have been based on the analytical or numerical resolution of the Eshelby's problem. They give access to the mean value of internal stress and energy in both phases, as a function of precipitate size and for different simple shapes chosen $a$ priori to be as close as possible to the observed ones [1-5]. However, they are generally limitated to simple precipitate shapes and low precipitate volume fractions.

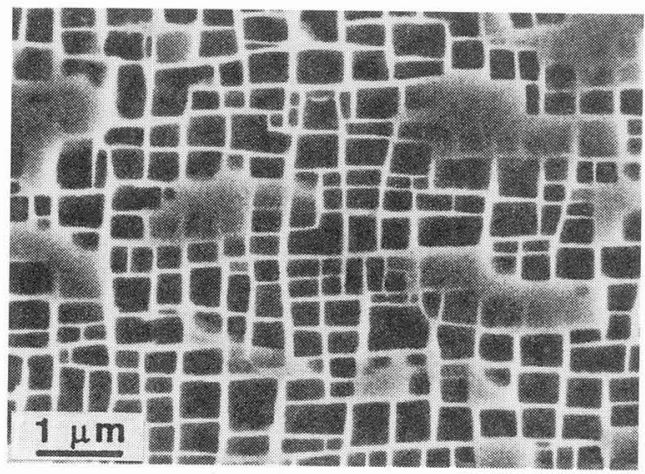

Figure 1: Typical microstructure of a single crystal Nibased superalloy (alloy "CMSX-2") after a standard heat treatment (3h. at $1315^{\circ} \mathrm{C}$; 5 h. at $1050^{\circ} \mathrm{C} ; 20 \mathrm{~h}$. at $870^{\circ} \mathrm{C}$ ). The single crystal is observed by S.E.M. in secondary electron mode, along one $\{001\}$ plane. The darker phase is $\gamma$ 'phase; the clearer one is $\gamma$.

The present paper proposes a synthesis of different recently published [6-9] or unpublished works in which a finite element approach of this problem was carried out. We estimate the internal stress and strain fields associated with a mismatching coherent precipitate, using a periodic $3 \mathrm{D}$ finite element model and 
assuming an anisotropic elastic behaviour for both $\gamma$ and $\gamma^{\prime}$ phases. Firstly, we compare the results of our model with data available from other analytical and numerical approaches. Then, we focus on the evolution of the local stress and strain fields as a function of precipitate volume fraction, which allows to point out the specificity of nickel-based superalloys in terms of internal stress distribution. Lastly, we discuss precipitate shape changes observed during precipitation and ageing heat treatments as well as during the early stage of a creep test.

\section{NUMERICAL MODELLING}

\subsection{Finite Element Model}

As a basic assumption, we consider an ideal $\gamma-\gamma^{\prime}$ alloy in which $\gamma^{\prime}$ precipitates are periodically distributed on a cubic array (Figure $2 a$ ). This allows to limitate the analysis to a cubic unit cell representative of the whole material. As illustrated in Figure $2 b$, this unit cell encloses one eighth of a single precipitate and one half of the surrounding matrix. Within this unit cell, different $\gamma-\gamma$, microstructures were modelled, using different $\gamma^{\prime}$ phase volume fractions and shapes. The $\gamma^{\prime}$ volume fractions tested ranged between $1 \%$ and $80 \%$. The different $\gamma^{\prime}$ shapes are illustrated in Figure 3. The cubic shape is close to the most current $\gamma^{\prime}$ morphology after standard heat treatment (cf Fig. 1). As it will be discussed in the following, "octet" and "star" morphologies are observed during the $\gamma$ ' precipitation process, while an evolution towards "infinite plate" morphology occurs during high temperature creep tests. The unit cells were discretized in finite element meshes such as the one showed in Figure $2 \mathrm{~b}$. In fact, the meshes varied with on the $\gamma-\gamma^{\prime}$ configuration but they were typically constituted of about 2500 elements. The lattice mismatch between $\gamma$ and $\gamma$ ' phases was introduced as an isotropic initial deformation of the $\gamma$ ' elements. In some cases, an uniaxial tension or compression stress parallel to the $\mathrm{Oz}$ direction was applied on the external x0y face of the elementary cell. Symmetry and periodicity were imposed by constraining three contiguous faces of the cell to deform along their initial plane, while the three other faces had to remain parallel to their opposite face. The mechanical behaviour of both $\gamma$ and $\gamma^{\prime}$ phases were assumed to be elastic and anisotropic.

Finite element calculations allow to obtain the strain and stress fields associated with a given precipitate morphology $\mathbf{M}_{\mathbf{k}}$ and a given volume fraction $\mathbf{V}_{\mathbf{v}}$. The internal elastic energy per volume of unit cell is deduced as :

$$
\mathcal{E}_{\mathrm{el} .}\left(\mathrm{M}_{\mathbf{k}}, \mathbf{V}_{\mathbf{v}}\right)=1 / 2 \Sigma_{1}\left[\sigma_{\mathrm{ij}}(\mathrm{l}) . \varepsilon_{\mathrm{ij}}(1)\right]
$$

where $\sigma_{\mathrm{ij}}(\mathrm{l})$ and $\varepsilon_{\mathrm{ij}}(1)$ are the $\mathrm{ij}$ components of the stress and strain tensors, calculated for each mesh element 1 of the unit cell. Note that analytical expressions of the elastic energy associated with precipitates with "cube", "octet" and "infinite plate" morphologies are available from the works of Khachaturyan et al. [2], provided that there is no interaction between precipitates (very low volume fraction), and that precipitate and matrix have the same elastic behaviour.

All calculations were performed using the finite element code SYSWELD [10].
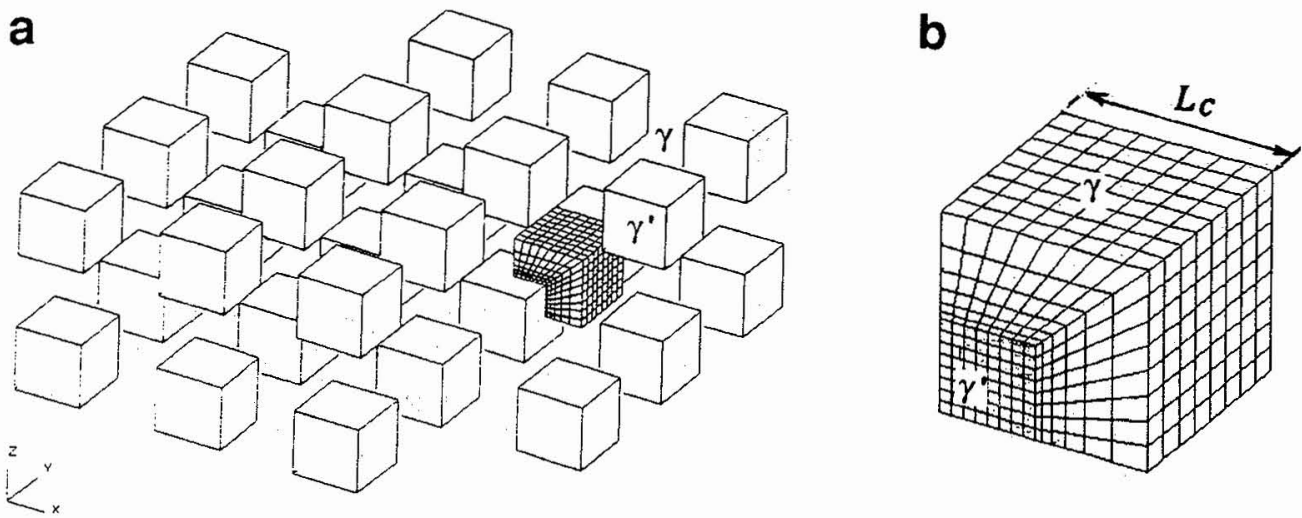

Figure 2: a) Ideal periodic distribution of precipitates ; b) unit cell used for the finite element calculations. The illustration is relative to a distribution of $10 \%$ of perfectly cubic precipitates. 

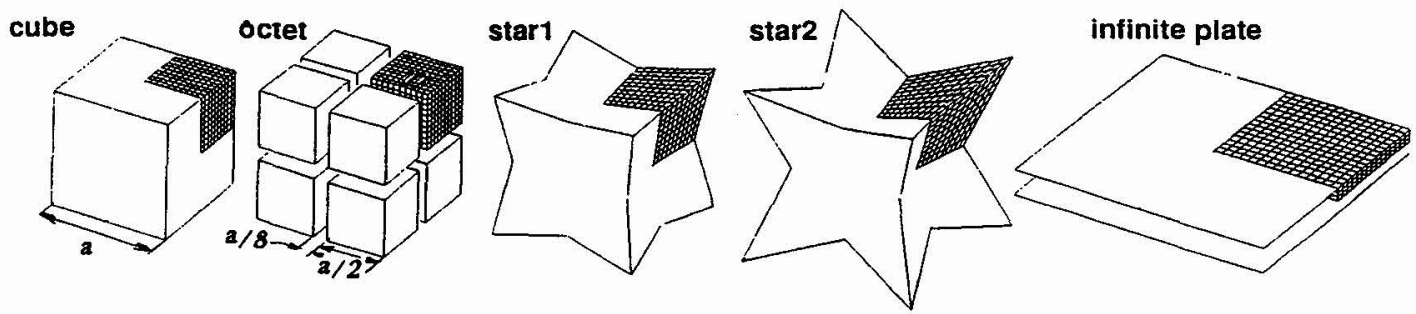

Figure 3: Different precipitate shapes tested in the present work. Crosshatched zones represent the eighth of the precipitate which is taken into account in the calculation cell. Lines are not related to the finite element mesh.

\subsection{Input data}

The discrepancy between $\gamma$ and $\gamma^{\prime}$ crystallographic lattices is usually quantified by the misfit parameter defined as $\delta=2\left(a^{\prime}-a\right) /\left(a^{\prime}+a\right)$, where $a$ and $a^{\prime}$ are the lattice parameters of $\gamma$ and $\gamma^{\prime}$ phases, respectively. In the case of industrial superalloys, $\delta$ is negative and ranges between about $-0.1 \%$ and $-0.5 \%$ at high temperature. A value of $-0.4 \%$ was used for the most part of the calculations reported in the present paper. Nevertheless, in the elastic case, stress levels are directly proportionnal to $\delta$ (and elastic energy to $\delta^{2}$ ), thus the conclusions presented in the following will remain qualitatively valid for other values of $\delta$, as far as the elastic assumption is supported.

The elastic constants of $\gamma$ and $\gamma^{\prime}$ phases were extracted from the literature. Experimental data relative to pure $\mathrm{Ni}$ or $\gamma$ model alloys and to pure $\mathrm{Ni} 3 \mathrm{Al}$ or $\gamma^{\prime}$ model alloys were analysed by two different ways. On one hand, the evolution of the Young's moduli along the $<001>$ directions, $\mathrm{E}_{001}$, was reported as a function of temperature (Figure 4; [6]). The Poisson's ratio $v$ and the anisotropy ratio $\mathbf{E}_{111} / \mathbf{E}_{\mathbf{0 0 1}}$ were supposed to be constant and equal for the two phases. Values of 0.35 and 2.4, respectively, were chosen from experimental results on $\gamma-\gamma^{\prime}$ superalloys. On the other hand, the evolution of $\mathrm{C}_{\mathrm{ij}}$ constants was directly reported as a function of temperature, as illustrated in Figure $5[8,9]$. As these two ways of analysing data lead to slightly different sets of elasticity constants, the $\mathrm{C}_{\mathrm{ij}}$ values used for the different cases treated in the following will be indicated at each time.

Whenever it was necessary to take into account the interfacial energy associated with a given microstructure, we assumed that $\gamma / \gamma^{\prime}$ interfacial tension $\sigma$ is isotropic. With regard to the large dispersion of $\sigma$ in the literature, we used a constant value of $14 \mathrm{~mJ} / \mathrm{m}^{2}$, which appeared to be intermediate in the experimental range.

Figure 4: Experimental temperature dependence of Young's modulus along the $<001\rangle$ direction, measured for pure $\mathrm{Ni}$, pure $\mathrm{Ni} 3 \mathrm{AL}$, and single crystals of different $\gamma$ and $\gamma$ model alloys (cf. $[6,8]$ for references; values in GPa)

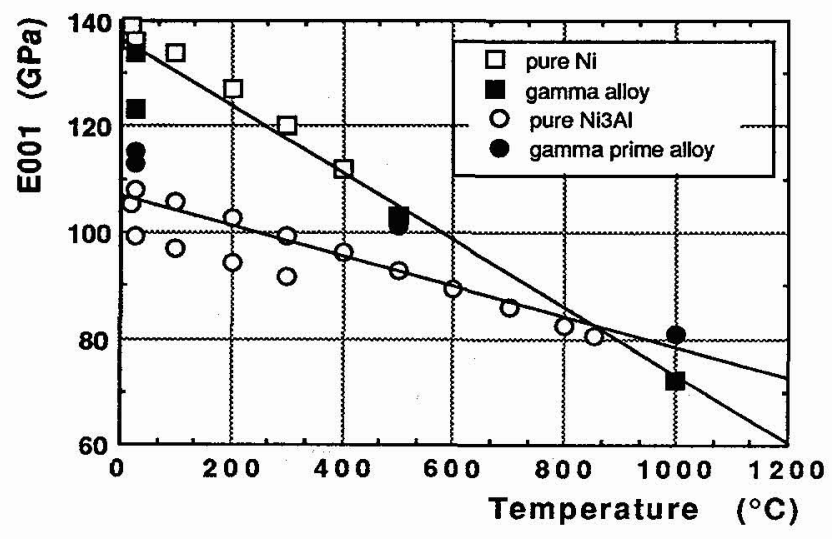



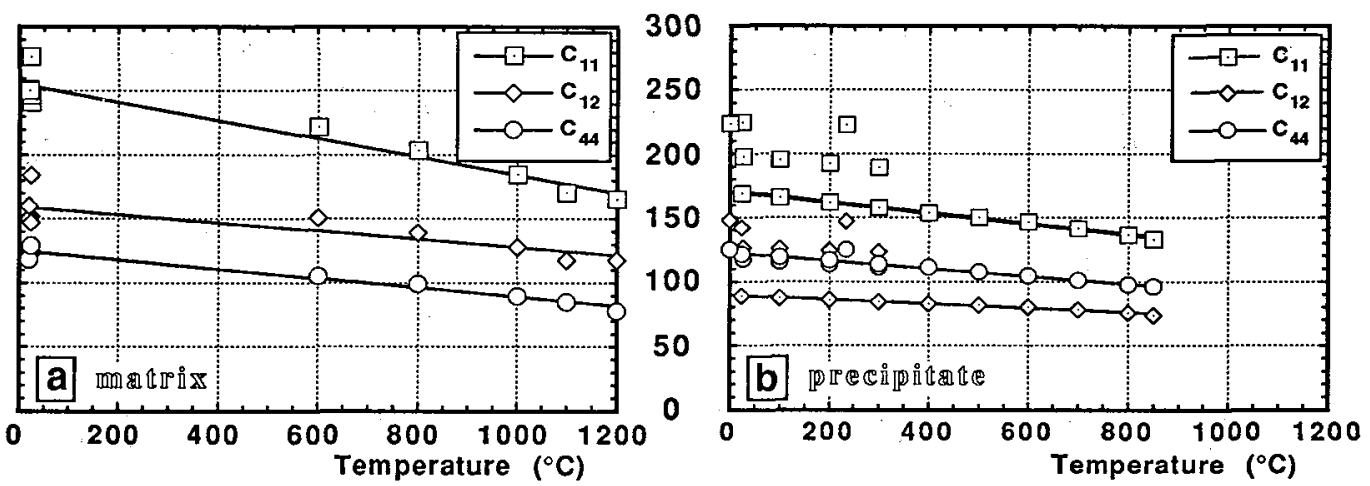

Figure 5: Experimental temperature dependence of elastic constants $\mathrm{C}_{\mathrm{ij}}$ (in GPa), in the case of pure Ni or $\gamma$ model alloys (a) and of pure NizAl or $\gamma$ model alloys (b) (cf. $[6,8]$ for references)

\section{RESULTS AND DISCUSSION}

\subsection{Comparison between different approaches}

Table 1 gives the values of elastic energy $\mathrm{E}_{\mathrm{el}}\left(\mathrm{M}_{\mathbf{k}}, 0.01\right)$ calculated in the case of a precipitate occupying 1 $\%$ of the elementary cell and presenting the different morphologies shown in Fig. 3. First line reports the results of analytical calculations obtained from the formulae proposed by Khachaturyan et al. [2], assuming that the two phases have the same elastic behaviour. Second and third lines concern finite element calculations, assuming that the two phases have the same or different elastic properties, respectively. Note that the values reported are relative to the elastic energy per volume of elementary cell. Two main comments can be made from Table 1. Firstly, the results of analytical and finite element approaches are in very good agreement. This proves that the discretization inherent in the finite element method does not lead to large bias in the estimation of elastic energy. Secondly, assuming different Cij coefficients for the two phases does not lead to drastically different results, at least for the set of input data used in our calculations. This confirms that the part of internal energy resulting from the difference of elastic moduli between the two phases can be reasonably neglected with regard to the other components, as assumed by Khachaturyan et al. and by other authors. In the following, the results will concern calculations with different properties for the two phases. The differences of elastic energy as a function of precipitate morphology will be discussed later.

Table 1: Elastic energy per volume of elementary cell associated with a mismatching precipitate $(\delta=-0.4 \%)$ occupying $1 \%$ of the elementary cell volume ( unit $=10^{4} \mathrm{~J} / \mathrm{m}^{3} ; \mathrm{C}_{11}=120 \mathrm{GPa}, \mathrm{C}_{12}=69 \mathrm{GPa}$ and $\mathrm{C}_{44}=85 \mathrm{GPa}$ for both $\gamma$ and $\gamma^{\prime}$ phases in lines 1 and 2 and for $\gamma^{\prime}$ phase in line $3 ; \mathrm{C}_{11}=169 \mathrm{GPa}, \mathrm{C}_{12}=121 \mathrm{GPa}$ and $\mathrm{C}_{44}=81$ GPa for $\gamma$ phase in line 3)

\begin{tabular}{|c|c|c|c|c|c|}
\cline { 2 - 6 } \multicolumn{1}{c|}{} & CUBE & OCTET & STAR 1 & STAR 2 & PLATE \\
\hline analyt. calcul. with $\mathrm{Cl}(\gamma)=\mathrm{Clj}\left(\gamma^{\prime}\right)$ & 2.562 & 2.401 & - & - & 1.754 \\
\hline F.E. calcul. with $\mathrm{Cij}(\gamma)=\mathrm{Cij}\left(\gamma^{\prime}\right)$ & 2.581 & 2.392 & 2.601 & 2.654 & 1.736 \\
\hline F.E. calcul. with $\mathrm{Cij}(\gamma) \neq \mathrm{Clj}\left(\gamma^{\prime}\right)$ & 2.556 & 2.365 & 2.579 & 2.635 & 1.738 \\
\hline
\end{tabular}

We also compared the results of our F.E. approach with those of a self-consistent model developed by the group of P. Franciosi in LPMTM, Villetaneuse, France [11]. In this model, the $\gamma-\gamma$ ' microstructure was idealized by an elementary symmetric pattern of several spheroids -seven in the example illustrated hereembedded in a mean medium. The $\gamma$ ' volume fraction was taken equal to $68 \%$, which is a typical value for superalloys after a standard heat treatment (cf Fig. 1). Different misfit values were tested, as well as different mono- and multiaxial loadings along the $<100\rangle$ directions. The results were compared in terms of 
mean values of the stress components in each phase. As an example, Table 2 shows the results obtained in the case of a misfit value of $-0.115 \%$ and of a biaxial loading along the $\mathrm{Oz}$ and $\mathrm{Oy}$ axes. The results of the two models are in very good agreement, the calculated values being always closer than $2 \mathrm{MPa}$ for all the tested conditions.

Table 2: Comparison between the mean values of $\sigma_{x}, \sigma_{y}$ and $\sigma_{z}$ stresses in $\gamma$ and $\gamma$ ' phases, calculated using either a Finite Element or a Self-consistent model (the two-phase material is loaded in biaxial tension of $100 \mathrm{Mpa}$ along $0 \mathrm{y}$ axis and $300 \mathrm{MPa}$ along $\mathrm{Oz}$ axis; $\delta=-0.115 \% ; \mathrm{C}_{11}=152 \mathrm{GPa}, \mathrm{C}_{12}=82 \mathrm{GPa}$ and $\mathrm{C}_{44}=100 \mathrm{GPa}$ for $\gamma$ phase and $\mathrm{C}_{11}=141 \mathrm{GPa}, \mathrm{C}_{12}=76 \mathrm{GPa}$ and $\mathrm{C}_{44}=93 \mathrm{GPa}$ for $\gamma^{\prime}$ phase). See [11] for more details.

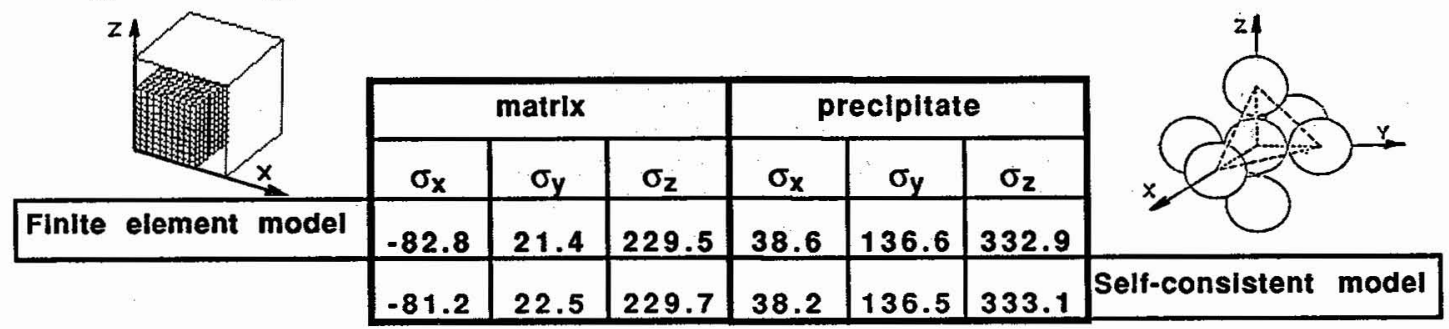

\subsection{Stress distribution in the standard microstructure}

Figure 6 shows the evolution of $\sigma_{\mathrm{x}}, \sigma_{\mathrm{y}}$ and $\sigma_{\mathrm{z}}$ stresses along the Ox axis of the elementary F.E. cell, for different volume fractions of precipitates with a cubic shape and a misfit of $-0.4 \%$ (same Cij values as in Table 1, third line). Note that along Ox axis these stress components are also the principal stresses. Fig. 6 points out the main specificity of the stress/strain field distribution in $\gamma-\gamma^{\prime}$ superalloys. In the most usual case of alloys strengthened by a relatively low volume fraction of coherent precipitates, the higher stress/strain levels are found within the precipitates and near the interface. Increasing the volume fraction of precipitates - without inverting the relative connectivity of the phases - leads to transfer the stress/strain maxima from the embedded precipitates to the embedding matrix.This is illustrated in an other way in Figure 7 which shows that increasing $\gamma$ ' volume fraction leads to increase the proportion of elastic energy stored in $\gamma$ phase. In the case of the standard microstructure of industrial superalloys illustrated in Fig. 1 (about $70 \%$ of cuboidal precipitates with a negative misfit at high temperature), the $\gamma$ channels appear to be highly stressed in biaxial compression, while $\gamma^{\prime}$ cuboids are stressed in isotropic tension but of a lower level (cf Figure 6 and 8).
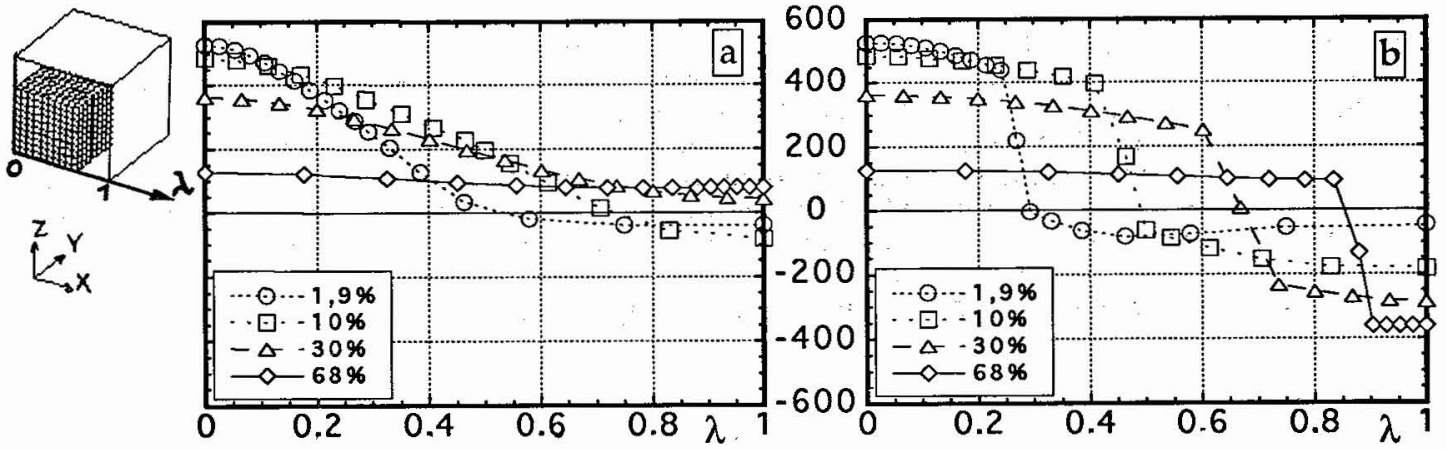

Figure 6: Evolution of $\sigma_{\mathrm{x}}$ (a), and $\sigma_{\mathrm{y}}$ and $\sigma_{\mathrm{z}}$ (b) stresses (expressed in MPa) along the Ox axis of the elementary F.E. cell, for different precipitate volume fractions $(\delta=-0.4 \%$; same Cij as in Table 1,3 rd row $)$

Several macro- and microscopical effects confirm this specific stress/strain distribution. The distortion of coherent $\gamma$ and $\gamma$ ' crystal lattices results in a broadening of X-ray, gamma-ray and neutron diffraction 
patterns, which hinder the measurement of lattice parameters. At the light of FE calculations, we showed that the difference in broadening of $\{002\}$ and $\{022\}$ gamma-ray diffraction peaks at high temperature is related to the high biaxial ("tetragonal") distortion of the $\gamma$ phase [7]. Müller et al. gave a similar interpretation to the broadening of neutron diffraction peaks [12]. At a microstructural scale, coherence stresses have also been associated with a shifting of "equilibrium" chemical composition of $\gamma$ and $\gamma$ ' phases [13]. The variations of stress level within $\gamma$ channels illustrated in Fig. 8 have also been related to the presence of chemical gradients $[14,15]$.

Internal stresses in $\gamma-\gamma$ ' superalloys noticeably influence their metallurgical and mechanical behaviour at high temperature. Some related microstructure evolutions will be discussed in the two next paragraphs. In the case of the standard microstructure, internal stresses are directly responsible for the alignment of the $\gamma$ ' cuboids along the $<001>$ crystal directions, as well as for the sharpening of their size distribution during isothermal "optimization" heat treatments. The periodic pattern of cubic precipitates with homogeneous size therefore appears to be a metastable "self-blocking" structure which has been shown to remain stable up to higher temperatures during a dissolution process [16,17]. In terms of mechanical behaviour, internal stress/strain fields interact with the dislocations which preferentialy move in the matrix phase at high temperature. Passing over the local stress maxima require a cooperative motion of several dislocations [18], which obviously has an additional strengthening effect.

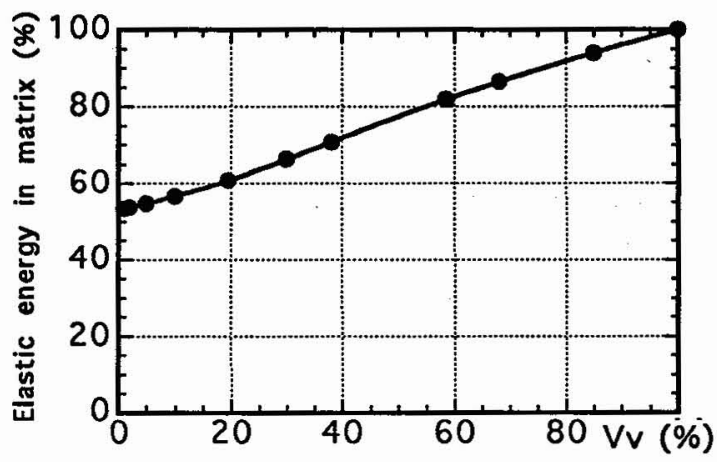

Figure 7: Proportion of elastic energy stored in $\gamma$ phase as a function of precipitate volume fraction

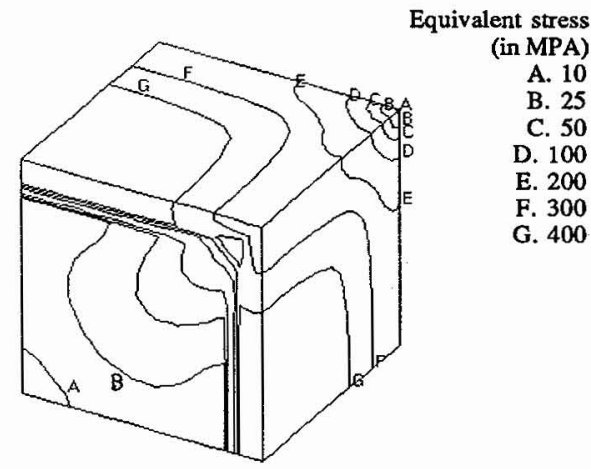

Figure 8: Distribution of the equivalent VonMises stresses resulting from lattice mismatch $(\delta=-0.4 \%$; same Cij as in Table 1,3rd row)

\subsection{Morphological evolutions during $\gamma^{\prime}$ precipitation}

In previous experimental works, we studied the $\gamma^{\prime}$ precipitation during continuous cooling from the $\gamma$ domain $[16,19]$. Microstructural observation of samples quenched from different temperatures during the precipitation process (between $1250^{\circ} \mathrm{C}$ and $1150^{\circ} \mathrm{C}$ ) showed that a growing precipitate encounters successive shape changes. In the case of a growth without interaction between precipitates (low nucleation density and low volume fraction), these changes follow the sequence "sphere $\rightarrow>$ cube $\rightarrow>$ octet $\rightarrow$ dendrites" illustrated in Figure 9. Sphere-to-cube and cube-to-octet transitions have been clearly related to the presence of internal mismatch stresses and to the anisotropic elastic behaviour of $\gamma$ and $\gamma$, phases [2,5]. In this paragraph, we will focus on the cube-to-octet transition, also denoted " $\gamma$ ' splitting". A typical microstructure illustrating this phenomenon is presented in Figure 10.

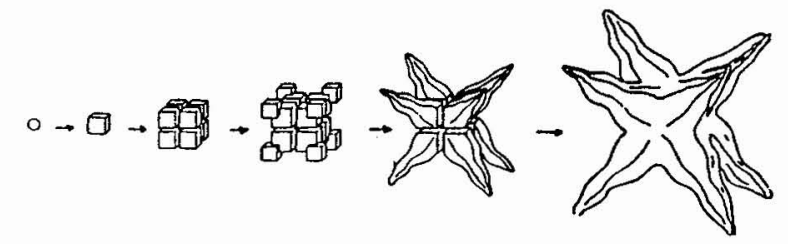

Figure 9: Schematization of the shape changes encountered by an isolated $\gamma$ ' precipitate during its coarsening or growth 


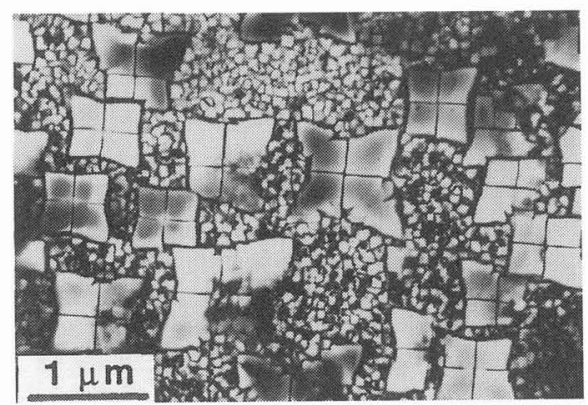

Figure 10: Microstructure observed in the case of a Nickel-based superalloy (AM1) quenched at $1225^{\circ} \mathrm{C}$ during a continuous cooling precipitation heat treatment (cooling rate $=-0.17^{\circ} \mathrm{C} / \mathrm{s}$ ). See $[16]$ for experimental details

Table 1 shows that splitting effectively leads to a decrease in elastic energy. This does not mean that this morphology should be the more stable, since the splitting of a cube also results in a doubling of $\gamma / \gamma$ ' interface area, i.e. in an increase of interfacial energy. As the mismatch elastic energy is proportional to the volume of the precipitate while the interfacial energy is proportional to its surface, it should be a critical precipitate size above which the splitting is favourable. This is confirmed by Figure 11a where the gain of total internal energy (elastic plus interfacial) resulting from splitting is plotted as a function of precipitate size. From an energetical point of view, the shape transition from cube to octet is expected as the precipitate coarsens. For a smaller precipitate size the "gain" appears to be negative, indicating that the compact single cube is stable because of the preponderance of interfacial energy. Above a critical cube size $\mathbf{a}_{\mathrm{c}->0}$ the gain of total energy corresponding to the transition from cube to octet becomes positive, indicating that the latter shape becomes more favourable. With our set of input data $(\delta=-0.4 \%$; same $\mathrm{Cij}$ as in Table $1,3 \mathrm{rd}$ line; $\sigma=14 \mathrm{~mJ} / \mathrm{m}^{2}$ ), we found that $\mathrm{a}_{\mathrm{c}->0}=0.44 \mu \mathrm{m}$.
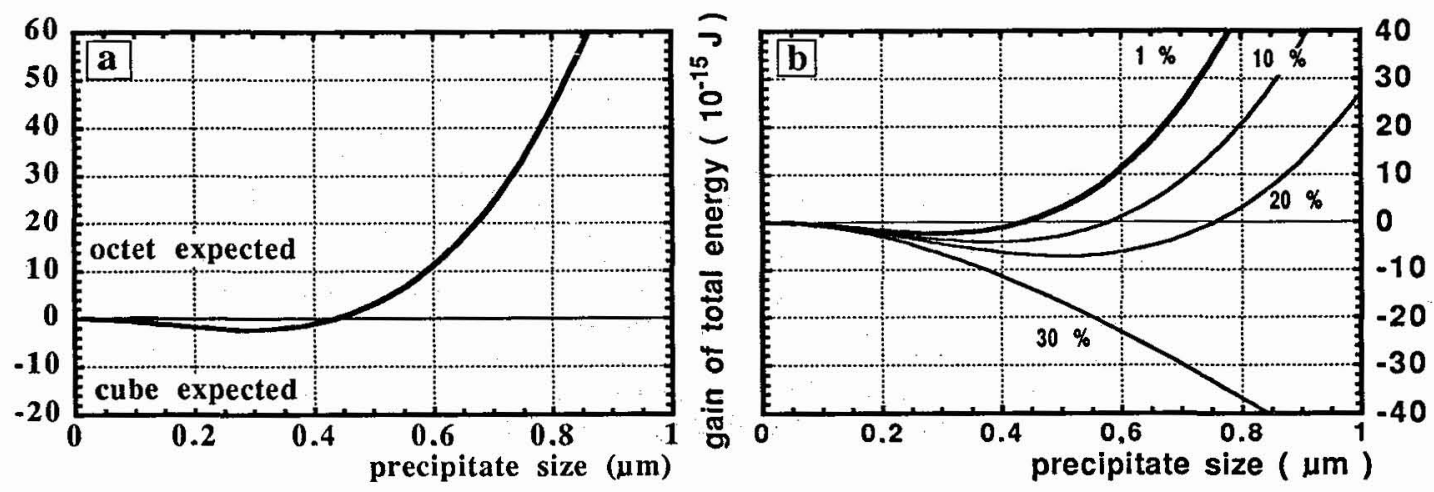

Figure 11: Evolution of the splitting energy of a coarsening precipitate as a function of its size: a) case of non interactive precipitates; b) case of interactive precipitates $\left(\delta=-0.4 \%\right.$; same Cij as in Table $1,3 \mathrm{rd}$ line; $\left.\sigma=14 \mathrm{~mJ} / \mathrm{m}^{2}\right)$

As F.E. approach allows to take into account the elastic interaction between precipitates, it is possible to study the evolution of $a_{c->0}$ as a function of precipitate volume fraction $V_{v}$. Calculations showed that increasing the volume fraction results in a dramatic decrease of the elastic driving force for splitting, that is in an increase of $a_{c->0}$. As illustrated in Figure $11 \mathrm{~b}, \mathrm{~V}_{\mathrm{V}}$ values equal to $10 \%, 19.5 \%$ and $30 \%$ led to $\mathrm{a}_{c-}$ $>0$ values of $0.59 \mu \mathrm{m}, 0.74 \mu \mathrm{m}$ and $2.55 \mu \mathrm{m}$, respectively ( for legibility purpose, the corresponding part of curve " $30 \%$ " is not represented ). Note that Fig. $11 \mathrm{~b}$ corresponds to the case of precipitates coarsening at constant volume fraction, that is during isothermal treatment. To describe a precipitation during continuous cooling, one has to suppose that all precipitates nucleate at the same time, with a nucleation density $\mathbf{N}_{\mathbf{V}}$. In this case, the precipitate volume fraction $V_{\mathbf{V}}$ is related to the precipitate size a by $V_{V}=$ $a^{3} N_{V}$. Figure 12 shows the evolution of the splitting energy during precipitate growth, as a function of 
precipitate size and for different values of $N_{\mathrm{V}}$. Note that the curve relative to a "free" growth in Fig. 12 is the same as the curve noted " $1 \%$ " in Fig. 11. Fig. 12 shows that the splitting phenomenon is expected to occur during precipitation as far as the nucleation density is not too high. In this case, the critical size ac->o is not very dependent on $\mathrm{N}_{\mathrm{v}}$, which results from the fact that the transition size is reached at low volume fractions of precipitated phase (typically lower than $10 \%$ ). As the nucleation density increases, a critical value is reached, above which the splitting never occurs. For the set of input data used in our calculations, the critical density was about $1.1 \mu \mathrm{m}^{-3}$ which corresponds to a distance between precipitate nuclei of 0.96 $\mu \mathrm{m}$. At higher values of $N_{V}$ the precipitate is expected to remain compact during the whole precipitation process. It is important to note that the non-occurrence of splitting is not related to a "geometric" effect ( when the critical size is higher than the distance available for growth ) but that it actually results from the long-range elastic interaction between neighbour precipitates. The agreement between these conclusions and experience is good, as discussed in details elsewhere $[8,9]$.

Figure 12: Evolution of the splitting energy of a growing precipitate as a function of its size and for different values of nucleation density $\mathrm{N}_{\mathrm{v}}$ $(\delta=-0.4 \%$; same $\mathrm{Cij}$ as in Table $13 \mathrm{rd}$ row; $\sigma$ $=14 \mathrm{~mJ} / \mathrm{m}^{2}$ )

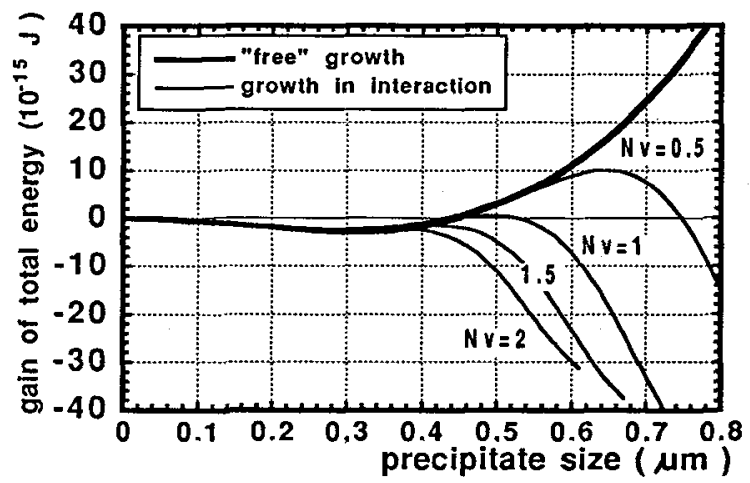

However, finite element calculations lead to conclude that the "star" morphology -an initially cubic precipitate elongated along the $<111>$ directions- is never expected to be favourable in terms of internal energy, since the cube-to-star evolution involves an increase of both elastic (cf. Table 1) and interfacial energies. Nevertheless, the typical distorded morphology illustrated in Fig. 10 is actually very often observed during the $\gamma^{\prime}$ precipitation process $[8,19,20]$. This disagreement between modelling and experiment is explained by the fact that the model does not take the kinetics aspect of the process into account, while the star morphology is clearly a transient stage of the shape evolution during precipitate growth. Using a model based on the microscopic diffusion and elasticity theories, Wang et al. [21] recently proved that the elongation of $\gamma^{\prime}$ cubes along the $<111>$ directions is a kinetic instability due to a preferential solute atom supply at the corners. In the same way, the evolution towards dendritic structures is clearly driven more by long range diffusion rather than by elastic accomodation.

\subsection{Morphological evolution during isothermal thermo-mechanical treatments}

Table 1 also shows that, from an elastic point of view, the "infinite plate" morphology is the more stable among the different shape tested. Khachaturyan et al. effectively noted that it is the shape of lowest elastic energy in the case of a couple matrix/precipitate with a negative elastic anisotropy [2]. In terms of total internal energy, the evolution towards plate morphology is expected if the volume fraction of precipitates is sufficiently high, typically higher than $30 \%$. This evolution is actually observed during long isothermal heat treaments at intermediate temperatures (around $1000^{\circ} \mathrm{C}$ ), the successive shapes changes during coarsening being described in this case by the sequence "sphere $\rightarrow$ cube $\rightarrow$ aligned cubes $\rightarrow$ plate" [8]. In this sequence, the "aligned cubes" structure is the optimum one previously discussed. We never observed the splitting phenomenon during ageing treatments at intermediate temperature, which supports the remarks of former paragraph, but desagrees which some propositions of the literature.

In the case of an homogeneous alloy submitted to an ageing treatment, the evolution towards plates occurs by equiprobable selection of one of the three available $<001>$ crystal directions, which leads to a homogeneous distribution of small platelets. However, any disturbance of the local cubic symmetry can promote one or two preferential orientations, which finally leads to a highly anisotropic structure of aligned 
rods or plates. As far as we know, this phenomenon, often denoted " $\gamma$ ' raftening", has been observed in the presence of two asymmetry causes. The first one is a large-scale chemical gradient, resulting either from segregation due to the solidification process [22] or from long range diffusion [23, 24]. In this case, the plates align perpendicular to the main chemical gradient [14]. The driving force for this phenomenon is not elucidated at the present time. The second one is an applied load, especially if loading direction is close to one $<001>$ crystal axis. Raftening under applied stress has been largely studied since it is known to occur in turbine blades during service. In this case, the direction of platelets depends on the direction and sign of the applied stress as well as of the sign of the $\gamma-\gamma^{+}$misfit. Typically, an alloy with a negative misfit will develop platelets perpendicular to a tensile applied stress and rods parallel to a compressive one, the evolution being inverted for a positive misfit, and no clear trend being detected for a misfit close to zero. This clearly shows that this phenomenon is related to the presence of mismatch stresses. However, its driving mechanisms are still in discussion (see [25-27] for a review). Actually, the main open question is to know if internal stresses are directly responsible for raftening, or if they induce it indirectly by way of guiding the creep plastic deformation within the matrix channels. In other words, is raftening able to occur in a purely elastic regime or does it require a preliminary plastic deformation ? In a recent experimental work, we acquired evidence for the first alternative to be possible [14]. Indeed, in the case of samples quenched in the very early stages of high-temperature low-stress creep tests, we detected preliminary signs for raftening (slight elongation of precipitates; chemical gradients between $\gamma$ channels) before evidence for creep deformation (dislocations in $\gamma$ channels). Figure 13 shows the stress fields calculated in the case of a $\gamma-\gamma^{\prime}$ standard structure submitted to an uniaxial tensile (13a) or compressive (13b) loading along the [001] direction, assuming that both phases deform elastically. It can be seen that the addition of internal and applied stresses effectively results in a local mechanical anisotropy. This anisotropy is supposed to be at the origin of shape evolution. This explanation is supported by the fact that inverting the stress direction (tension or compression) or the misfit sign leads to invert the direction of stress/strain gradient. However, at the present time, the kinetics aspect of this stress-induced shape evolution cannot be treated in our approach, for at least two reasons, a technical and a theoretical one. Firstly, as illustrated in the previous paragraphs, the current finite element approach only allows to compare stress/strain states between different microstructures. To describe the continuous evolution from one microstructure to another, it has to be associated with a kinetics approach such as Monte-Carlo (M.C.) method (see [28] for an example of combination of F.E. with M.C. approaches). Secondly, the basic mechanisms of the coupling between stress/strain fields and shape evolution are still not well known. This coupling can be considered to take place through a stress-induced diffusion mechanism, through the selection of preferential interfaces during coarsening process, through a local modification of the equilibrium state of the two-phase material, etc... The choice of a shape evolution criterion will obviously require a better knowledge of the mechanism(s) which drive(s) the raftening.
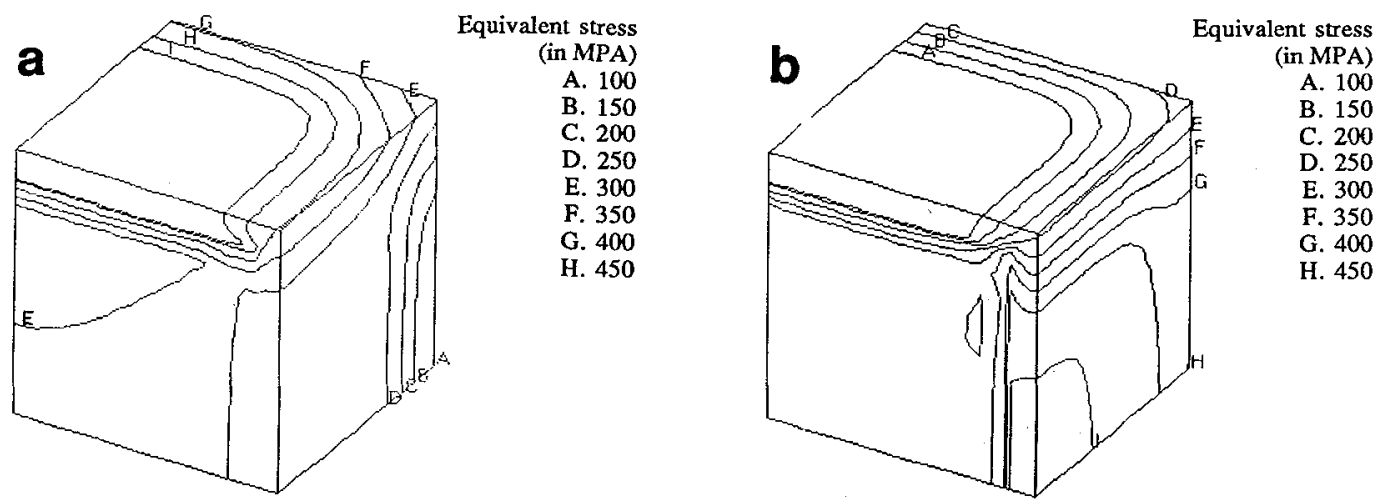

Figure 13: Distribution of the equivalent VonMises stresses in the case of a single-crystal superalloy submitted to a tension (a) or compression (b) stress of $300 \mathrm{MPa}$ along $\mathrm{Oz}$ direction $\left(\delta=-0.3 \% ; \mathrm{C}_{11}=109 \mathrm{GPa}, \mathrm{C}_{12}=59 \mathrm{GPa}\right.$ and $\mathrm{C}_{44}=72$ $\mathrm{GPa}$ for $\gamma$ phase and $\mathrm{C}_{11}=125 \mathrm{GPa}, \mathrm{C}_{12}=67 \mathrm{GPa}$ and $\mathrm{C}_{44}=82 \mathrm{GPa}$ for $\gamma^{\prime}$ phase) 


\section{CONCLUSIONS}

Finite element micromechanical modelling was shown to be a powerful tool for analyzing the morphological changes encountered by coherent precipitates under the influence of mismatch stresses. It gives access to the stress/strain fields and to the internal energy associated with various morphologies and different volume fractions of precipitation. Thus, on the basis of an energetical criterion, it becomes possible to determine the precipitate shapes expected to be observed during a near-equilibrium thermomechanical treatment, and to study the influence of material properties (mismatch, interfacial tension, elastic anisotropy ) and of treatment parameters (temperature, applied stress or strain, cooling or heating rate ...) on this microstructural evolution. Its major limitations are found in the lack of accurate data for the parameters required for the calculation, but above all in the fact that it does not take into account the kinetics angle of the evolution process.

\section{Acknowledgements}

The present study was performed in the frame of the french national research programme " Contrat de Programme de Recherche sur la Stabilité Structurale des Superalliages Monocristallins "

\section{References}

[1] Pineau A., Acta metall. 24 (1976) 559-564

[2] Khachaturyan A.G., Semenovskaya S.V and Morris J.W.Jr, Acta metall. 36 (1988) 1563-1572

[3] Doi M, Miyazaki T. and Wakatsuki T., Mat. Sci. Engin. 67 (1984) 247-253

[4] Miyazaki T., Doi M. and Kozakai T., Diff. defect data,Solid state phen. 3\&4 (1988) 227-236

[5] Müller L., Glatzel U. and Feller-Kniepmeier M., Acta metall. mater. 40 (1992) 1321-1327

[6] Ganghoffer J.F., Hazotte A., Denis S.and Simon A., Scripta metal. mater. 25 (1991) 2491-2496

[7] Hazotte A., Bellet D., Ganghoffer J.F., Denis S, Bastie P. and Simon A., Phil. Mag. Letters 66 (1992) 189-196

[8] Hazotte A. and Denis S., "Calculation of internal mismatch stresses related to $\gamma$ ' precipitate morphology in Nickel-based superalloys", 4th Int. Conf. on Residual Stresses (ICRS4), Baltimore (U.S.A.), june 8-10, 1994, (The Society for Experimental Mechanics) pp. 825-833

[9] Hazotte A., Grosdidier T., Denis S., submitted to Scripta metall. mater

[10] SYSWELD Userst Manual - Framasoft+C.S.I

[11] Estevez R., Franciosi P., A. Hazotte A. and Denis S., "Phase inhomogeneities in deformed superalloy single crystals: Numerical estimates", Conf. IUTAM'94, Nottingham (G.B.), sept.1994

[12] Müller L., Link T.and Feller-Kniepmeier M., Scripta metall. mater. 26 (1992) 1297-1302

[13] Miyazaki T., Koyama T and Doi M., Acta metall. mater. 40 (1994) 3417-3424

[14] A. Racine, Thèse de 1 "Institut National Polytechnique de Lorraine" (INPL), Nancy FRANCE (1995), to be presented

[15] Racine A. and Hazotte A., J. de Phys. IV, supp. J. de Phys. III 3 (1993) 355-358

[16] T. Grosdidier, Thèse de I"Institut National Polytechnique de Lorraine" (INPL), Nancy FRANCE (1992)

[17] Grosdidier T., Hazotte A. and Simon A., Scripta metall. mater. 30 (1994) 1257-1262

[18] Coujou A.and Clement N., "Déformation "in-situ" de superalliages monocristallins", Nat. French Col. "Superalliages Monocristallins", Toulouse-Seilh FRANCE, march 22-24, 1995, proceedings to be published

[19] Ricks R.A., Porter A.J and Ecob R.C., Acta metall. 31 (1983) 43-53

[20] Maheshwari A.and Ardell A.J., Scripta metall. mater. 26 (1992) 347-352

[21] Wang Y. and Khachaturyan A.G., Scripta metall. mater. 31 (1994) 1425-1430

[22] Hazotte A. and Lacaze J., Scripta metall. 23 (1989) 1877-1882

[23] T. Massard T., Morrison A., Koutny J.L. and Bienvenu Y., Proc. "4èmes Journées Nat. du Soudage", (1989) pp 164

[24] Draper S., Hull D. and Dreshfield R., Metall. Trans. A 20A (1989) 683-688

[25] Pollock T.M., Argon A.S., Acta metall. mater. 42 (1994) 1859-1874

[26] Socrate S, Parks D.M., Acta metall. mater. 41 (1993) 2185-2209

[27] Louchet F., "La coalescence orientée sous contrainte", Nat. French Col. "Superalliages Monocristallins", Toulouse-Seilh FRANCE, march 22-24 (1995), proceedings to be published

[28] Gayda J., Srolowitz D.J., Acta Metall. 37 (1989) 641-650 